\title{
The prognostic impact of $P$ wave dispersion in prediction of clinical outcome after percutaneous balloon mitarl valvuloplasty in patients with mitral stenosis and sinus rhythm
}

\author{
Ragab A. Mahfouz, Ashraf E. Dewedar
}

Department of Cardiology, Faculty of Medicine, Zagazig University, Zagazig, Egypt

Email: ashrafdwedar@yahoo.com

Received 25 January 2012; revised 31 March 2012; accepted 29 April 2012

\begin{abstract}
Objective: The aim of the study was to investigate the impact of P-maximum and P-wave dispersion on the long term clinical outcome after successful percutaneous balloon mitral valvuloplasty (PBMV) in patients with mitral stenosis (MS) and sinus rhythm. Also to test the correlation between P-variables and right ventricular function and pulmonary artery pressure before and after PMBV. Methods: Eighty-five patients undergoing PMBV were enrolled in this study. We evaluated P-maximum, P-minimum and P-wave dispersion before and one month after PBMV. We studied the changes in pulmonary arterial pressure (PAP), left atrial (LA) dimension, mitral diastolic gradient, and mitral valve area, in addition to the changes in right ventricular function utilizing tissue Doppler assessment both before and after PMBV, in addition the role of the $P$-wave dispersion in prediction of late cardiac events. Results: There were significant decreases in mean diastolic gradient, PAP, and $L A$ size and significant improvement in right ventricular tissue Doppler indices after PMBV. Accompany these hemodynamic changes after PMBV. $P$-maximum and $P$-wave dispersion were found to be decreased $(P<0.001)$. Patients developed cardiac events during follow-up had a higher P-maximum and P-dispersion than those without late cardiac events $(P<0.001)$. Moreover the changes in $P$-maximum and P-dispersion before and after PMBV in patients with cardiac events were not significant, while $\mathbf{P}$-maximum and $\mathbf{P}$-dispersion significantly $(\mathrm{P}<$ 0.002) decreased in patients without events It was revealed with linear regression and correlation analysis that the degree of and the changes in P-maximum and $P$-wave dispersion were correlated with development of late cardiac events after PMBV, with Cut-
\end{abstract}

off values of $\geq 62.8$ msec for $P$-wave dispersion and 118 mes for P-maximum.ROC curve showed AUC of 0.919 for P-wave dispersion and 0.913 for P-maximum $(P<0.001)$. Conclusion: $P$-wave maximum and dispersion are significantly increased in patients with mitral stenosis. These changes decreased significantly after PMBV. The P-maximum and P-wave dispersion changes were correlated with significant impairment of right dysfunction and the degree of pulmonary artery pressure. $\mathrm{P}$-maximum and $\mathrm{P}$-wave dispersion could be considered as independent predictors of late outcome of patients with MS after successful PMBV (AF, recurrent hospital admission, embolic phenomenon deterioration of right ventricular function).

Keywords: P-Wave Maximum P-Wave Minimum; P-Wave Dispersion; Rheumatic Mitral Stenosis; Cardiac Events; Follow-Up

\section{INTRODUCTION}

$\mathrm{P}$-wave prolongation has been used as a marker of interatrial conduction disturbance and prolonged atrial conduction time, which has been associated with a high frequency of atrial fibrillation (AF). These atrial conduction disturbances are nonuniform and site dependent, and may result in a highly variable P-wave duration in the different leads of a 12-lead electrocardiogram (ECG). As a marker of this variation, the P-wave dispersion has been introduced as the difference between the maximum and minimum P-wave duration. Prolonged P-wave duration and $\mathrm{P}$-wave dispersion have been found to be predictive of later $\mathrm{AF}$ in patients with idiopathic $\mathrm{AF}$ [1-3], and recurrence of AF following cardioversion $[4,5]$.

Although there are numerous predictors of late events following the procedure - age, functional class IV, previous surgical commissurotomy, moderate to severe mitral 
deficit, echo score $>8$, severe mitral deficit following dilatation and increased pressure in the pulmonary artery after dilatation, small valve area after the procedure $\left(<1.5 \mathrm{~cm}^{2}\right)$, non-sinus rhythm, valve calcification on fluoroscopy, increased LA pressure, increased transvalvular gradient [6-11]. Two case control studies identified a significant decrease in $\mathrm{P}$ wave indices at one month following mitral balloon valvuloplasty for mitral stenosis, and there is less understanding about the meaning of these changes at late follow-up after the percutaneous intervention $[12,13]$. Even there are no studies about the impact of $\mathrm{P}$-wave duration and $\mathrm{P}$-wave dispersion in prediction of late events after percutaneous balloon mitral valvuloplasty.

\section{AIM OF THE STUDY}

The aim of the study was to investigate the impact of P-maximum and P-wave dispersion on the long term clinical outcome after successful PBMV in patients with mitral stenosis (MS) and sinus rhythm. Also test the correlation between $\mathrm{P}$-maximum and $\mathrm{P}$-dispersion to right ventricular function and pulmonary artery pressure before and after PMBV.

\section{SUBJECTS AND METHODS}

A total of 85 patients with mitral stenosis and fulfill the criteria needed for percutaneous mitral balloon valvuloplasty were included in the study. Only persons 35 years of age or younger were included in the study to minimize the chance of the presence of associated coronary artery disease. Inclusion criteria: 1) pure mitral stenosis of rheumatic origin; 2) nonexistent or mild mitral insufficiency; 3) absence of concomitant hemodynamically significant other valvular disease; 4) TDI-derived good quality echocardiographic imaging allowing measurements of tricuspid annular velocities; 5) absence of any disease that could affect myocardial function (e.g. coronary artery disease, chronic lung disease, cardiomyopathies); 6) absence of atrioventricular conduction abnormalities and atrial fibrillation. They were classified into two groups according to the development of cardiac events along the period of follow-up. Group I, included those with cardiac events and group II included those without cardiac events during follow-up.

Sixty two age- and sex-matched healthy subjects were also enrolled as the control group.

\subsection{PBMV Procedure}

All procedures were performed by the anterograde transseptal approach. A double balloon was used in 85 patients with mitral stenosis on sinus rhythm, according to the stepwise technique, under fluoroscopy guidance. A successful PBMV, was defined as an MVA $\geq 1.5 \mathrm{~cm}^{2}$ or $\geq 1 \mathrm{~cm}^{2} / \mathrm{m}^{2}$ with no regurgitation $>2 / 4$.

\subsection{P-Wave Dispersion Measurement}

$\mathrm{P}$ wave duration was measured in all simultaneously recorded 12 leads of the surface ECG. All recordings were performed in the same quiet room during spontaneous breathing, following 20 minute of adjustment in the supine position. P-wave indices were evaluated before mitral valvuloplasty and one month after. $\mathrm{P}$ wave duration measurements were obtained manually using calipers and magnifying lens for accurate definition of the ECG deflection as defined in a previous study [14]. The onset of the $\mathrm{P}$ wave was defined as the point of the first visible upward departure of the trace from the bottom of the baseline. The return to the baseline of the bottom of the trace in wave was considered to be the end of the $\mathrm{P}$ wave. $P$ maximum in any of the 12 lead surface ECGs was measured and used as a marker of prolonged atrial conduction time. The difference between P-wave maximum and $\mathrm{P}$-wave minimum durations was defined as $\mathrm{P}$-wave dispersion.

\subsection{Echocardiography}

Echocardiographic examination was performed using available transthoracic ultrasound equipment (HB-5500, with a phased-array transducer of $2.5 \mathrm{MHz}$ ). Echocardiographic examination, including M-mode, two-dimensional (2D), pulsed and color Doppler measurements and TDI parameters were recorded before PBMV and analyzed off-line. Measurements were done and evaluated according to recent guidelines 15 .

M-mode study was used to measure the left atrial dimension. To determine the transmitral valve gradient, four-chamber apical view was used with the sample volume placed at the mitral valve coaptation point. Transmitral valve gradient was defined as the mean of gradients obtained from three consecutive beats obtained by continuous wave Doppler study. Mitral valve area was measured by planimetry of the mitral valve in short axis view and the mean of three measurements in three different beats were accepted as final. Mitral valve areas of $1 \mathrm{~cm}^{2}$ or less were considered to be very severe, $1 \mathrm{~cm}^{2}$ to $1.5 \mathrm{~cm}^{2}$ were considered to be moderate and greater than $1.5 \mathrm{~cm}^{2}$ were considered to be mild cases.

Colour flow Doppler was used to detect the presence of associated valvular insufficiencies, which were additionally confirmed by Doppler studies. The maximal tricuspid regurgitation (TR) velocity was recorded by continuous-wave Doppler and pulmonary artery systolic pressure (PASP) was calculated using the modified Bernoulli equation, after estimating right atrial pressure (RAP) with a method developed by Lichtenstein [15]. Values of PASP $\geq 35 \mathrm{mmHg}$ defined pulmonary hypertension. The 
$\mathrm{RV}$-diameter was measured in the apical four-chamber view above the tricuspid annular plane. RV function was assessed by, tricuspid annular plane systolic excursion (TAPSE). TAPSE as a parameter for RV long axis function was assessed with M-Mode cursor positioned at the free wall angle of the tricuspid valve annulus, whereas data were averaged over five beats as it has been recommended [16]. Pulsed Doppler tissue imaging techniques (TDI) were used for estimation of tricuspid annular systolic and diastolic velocities before and in the next day after PMBV, after placing the cursor at the junction of the right ventricle free wall and the anterior leaflet of the tricuspid valve, using the 2D four-chamber view. The peak systolic $(\mathrm{Sm})$, peak early diastolic $(\mathrm{Em})$, and peak late diastolic (Am) annular velocities were calculated and averaged over three consecutive beats, as it has been previously described [17].

Intra- and inter-observer measurement errors for all the manually measured ECG were 2.7 and 2.9 for Pwave Dispersion and 2.2 and 2.3 for P-maximum

While the Interobserver and intraobserver variability in these measurements which can be relatively high, so our Doppler by 2 investigators. There were minor intraobserver variations; $1.00 \% \pm 1.35 \%$ for $\mathrm{MV}$ area, $2.74 \%$ $\pm 0.15 \%$ for LA diameter. $2.1 \%+0.9 \%$ for mitral pressure gradient, $1.1 \%+0.32 \%$ for $\mathrm{Sm}$ and $0.3+0.11$ for MV score.

\subsection{Follow-Up}

All patients were followed-up for a mean period of $48+$ 11 months. All patients were evaluated by complete echocardiographic study after one month following balloon mitral valvuloplasty. The follow-up visits after that were performed every 6 months and upon any symptoms like dyspnea or palpitation. The follow-up studies included complete history taking and thorough clinical examination, electrocardiogram as well as complete echo-Doppler assessment.

\section{STATISTICAL ANALYSIS}

All the analyses were performed using a commercially available package (SPSS, Rel. 11.0, 2002, SPSS Inc., Chicago). To compare MS patients vs. references or MS patients with events vs MS patients without events after PBMV, the unpaired Student's $t$-test was performed for continuous variables, whereas for categorical variables, Fisher's exact test was used. Quantitative values are presented as mean \pm 1 standard deviation (SD). Relation between quantitative variables was studied using the linear regression. In the search for a diagnostic cut-off value for occurrence of events at follow-up, a receiver operating characteristic (ROC) curve analysis was constructed and the area under the curve is reported, which is representa- tive of the discriminatory ability of the variable cut-off. Sensitivity and specificity, positive and negative predictive values of the best cut-off variable were calculated. A P-value of $<0.05$ was considered statistically significant $[18,19]$.

\section{RESULTS}

A total of 85 patients with mitral stenosis and fulfill the criteria needed for percutaneous mitral balloon valvuloplasty were included in the study. They were classified into two groups according to the development of cardiac events along the period of follow-up. One group with cardiac events and another group without cardiac events. All patients were in sinus rhythm. After $(48+11)$ months period of follow-up, $27(31.8 \%)$ patients out of 85 had events: 22 patients developed persistent AF, 4 patients became symptomatic (NYHA class III and occurrence of symptoms with pulmonary pressure $>75 \mathrm{mmHg}$ ), one underwent percutaneous valvuloplasty for restenosis), and two cases developed stroke (associated with recent paroxysmal AF).

Table 1 compares the basic demographic and echocardiographic variables of patients with mitral stenosis and control group. The echocardiographic variables before and after one month of the procedure are compared in Table 2. The P-wave variables were significantly higher in patients with mitral stenosis compared to control subjects Table 3.

Table 4 shows that the P-wave variables significantly decreased after successful PMBV.

\section{PREDICTION OF CARDIAC EVENTS AFTER PBMV}

Maximum P-wave duration and PWD of patients were significantly higher in patients with cardiac events than in those without events during the period of follow-up, both before and after PBMV $(\mathrm{P}<0.001$ and $\mathrm{P}<0.001$, respectively) Table 5. However, there was no statisticcally significant difference between the two groups regarding minimum $\mathrm{P}$-wave duration $(\mathrm{P}>0.05)$. Table 6 represents a significant correlation between the P-wave dispersion and the degree of pulmonary hypertension and tissue Doppler indices of impaired right ventricular dysfunction.

ROC analysis revealed that the best cut-off value of P-wave dispersion of $\geq 62.8 \mathrm{msec}$ separate patients with from those without events during follow-up, with a sensitivity of $89.2 \%$, specificity of $96.4 \%$, positive predictive value of $92.5 \%$ and negative predictive value of 98.2\% (AUC $=0.919, \mathrm{P}<0.001)$. ROC analysis revealed that $\mathrm{P}$-maximum of $\geq 118 \mathrm{msec}$ separate patients with from those without events during follow-up with a sensitivity of $85.5 \%$, specificity of $91.8 \%$, positive predictive 
Table 1. General characteristics of the studied samples.

\begin{tabular}{|c|c|c|c|}
\hline & MS patients $(\mathrm{n}=\mathbf{8 5})$ & Reference $(n=62)$ & P-value \\
\hline Age (years) & $28 \pm 6$ & $27 \pm 2$ & $>0.05$ \\
\hline $\operatorname{Sex}(M / F)$ & $64 / 21$ & $23 / 19$ & $>0.05$ \\
\hline $\operatorname{BSA}\left(\mathrm{m}^{2}\right)$ & $1.65 \pm 0.3$ & $1.66 \pm 0.3$ & $>0.05$ \\
\hline Heart rate (bpm) & $78 \pm 12$ & $76 \pm 11$ & $>0.05$ \\
\hline Systolic blood pressure (mmHg) & $117 \pm 11$ & $119 \pm 12$ & $>0.05$ \\
\hline Diastolic blood pressure (mmHg) & $70 \pm 7$ & $72 \pm 7$ & $>0.05$ \\
\hline Echo-score & $7.35 \pm 2.1$ & - & \\
\hline MVA $-2 D$ planimetry $\left(\mathrm{cm}^{2}\right)$ & $0.95 \pm 0.08$ & - & \\
\hline MVA-PHT $\left(\mathrm{cm}^{2}\right)$ & $1.03 \pm 0.11$ & - & \\
\hline Mean transmitral gradient $(\mathrm{mmHg})$ & $31.52 \pm 8.7$ & - & \\
\hline LA antero-posterior diameter $(\mathrm{mm})$ & $48.1 \pm 2.9$ & $31 \pm 2$ & $<0.0001$ \\
\hline LVEDD (mm) & $42.3 \pm 3.1$ & $45.6 \pm 2.2$ & $>0.05$ \\
\hline $\operatorname{LVESD}(\mathrm{mm})$ & $25.1 \pm 1.9$ & $28 \pm 2.1$ & $>0.05$ \\
\hline LVEF $(\%)$ & $76.3 \pm 5.5$ & $77.9 \pm 6.2$ & $>0.05$ \\
\hline $\mathrm{RV}$ diastolic diameter (mm) & $26.85 \pm 1.2$ & $19 \pm 2$ & $<0.05$ \\
\hline PASP (mmHg) & $64.05 \pm 8.61$ & $18 \pm 3$ & $<0.0001$ \\
\hline TAPSE & $14.15 \pm 1.4$ & $23.9 \pm 3.2 \mathrm{~mm}$ & $<0.003$ \\
\hline Sm & $10.41 \pm 1.2$ & $16.19 \pm 1.0$ & $<0.001$ \\
\hline Em/Am ratio & $1.19 \pm 0.13$ & $2.01 \pm 0.15$ & $<0.001$ \\
\hline
\end{tabular}

BSA = body surface area, MVA = mitral valve area, $2 \mathrm{D}=$ two dimensional, $\mathrm{PHT}=$ Pressure half time, $\mathrm{LVEDD}=$ left ventricular end diastolic, $\mathrm{LVESD}=$ left ventricular end systolic, PASP = pulmonary artery systolic pressure, TAPSE = Tricuspid annular plan systolic excursion, Sm: systolic tricuspid myocardial velocity; E/Em: ratio of early diastolic transtricusid flow velocity to early diastolic peak myocardial velocity.

Table 2. Echocardiographic data before and immediately after percutaneous mitral valvuloplasty (PTMV).

\begin{tabular}{|c|c|c|c|}
\hline Variable & Before & After & P-value \\
\hline LA antero-posterior diameter $(\mathrm{mm})$ & $48.1 \pm 2.9$ & $43.1 \pm 2.3$ & $<0.0001$ \\
\hline LVEDD $(\mathrm{mm})$ & $42.3 \pm 3.1$ & $43.2 \pm 5.1$ & $>0.05$ \\
\hline $\operatorname{LVESD}(\mathrm{mm})$ & $25.1 \pm 1.9$ & $27.04 \pm 3.9$ & $>0.05$ \\
\hline LVEF (\%) & $76.3 \pm 5.5$ & $73.9 \pm 9.2$ & $>0.05$ \\
\hline $\mathrm{RV}$ diastolic diameter $(\mathrm{mm})$ & $26.85 \pm 1.2$ & $22.8 \pm 1.5$ & $<0.01$ \\
\hline $\operatorname{MVA}\left(\mathbf{m m}^{2}\right)[$ Planimetry] & $0.95 \pm 0.08$ & $1.83 \pm 0.23$ & $<0.001$ \\
\hline $\operatorname{MVA}\left(\mathrm{mm}^{2}\right)[\mathrm{PHT}]$ & $1.03 \pm 0.11$ & $1.75 \pm 0.17$ & $<0.001$ \\
\hline Mean transmitral gradient $(\mathrm{mmHg})$ & $17.7 \pm 3.2$ & $4.3 \pm 1.7$ & $<0.0001$ \\
\hline Maximum transmitral gradient $(\mathrm{mmHg})$ & $31.6 \pm 8.3$ & $7.2 \pm 3.3$ & $<0.0001$ \\
\hline PASP (mmHg) & $72.8+18.30$ & $28.9 \pm 11.2$ & $<0.001$ \\
\hline TAPSE & $14.11 \pm 2.1$ & $22.3 \pm 2.1$ & $<0.03$ \\
\hline Sm & $10.9 \pm 1.1$ & $14.6 \pm 1.7$ & $<0.02$ \\
\hline Em/Am ratio & $1.12 \pm 0.14$ & $1.54 \pm 0.17$ & $<0.05$ \\
\hline
\end{tabular}

MVA = mitral valve area, PHT = pressure half time, LVEDD = left ventricular end diastolic, LVESD = left ventricular end systolic, PASP = pulmonary artery systolic pressure, $\mathrm{LVEF} \%$ = Left ventricular ejection fraction $\%$, TAPSE = Tricuspid annular plan systolic excursion, Sm: systolic tricuspid myocardial velocity; E/Em: ratio of early diastolic transtricusid flow velocity to early diastolic peak myocardial velocity. 
Table 3. P-wave variables among patients with mitral stenosis versus control subjects.

\begin{tabular}{cccc}
\hline Variable & Patients with MS & Control subjects & P-value \\
\hline P-wave maximun & $128.7 \pm 19.6$ & $84.5 \pm 12.9$ & $<0.001$ \\
P-wave minimum & $63.3 \pm 8.6$ & $46.2 \pm 7.3$ & $<0.001$ \\
P-wave dispersion & $65.2 \pm 5.7$ & $38.1 \pm 4.2$ & $<0.001$ \\
\hline
\end{tabular}

Table 4. P-wave variables among patients with mitral stenosis before and after PBMV.

\begin{tabular}{cccc}
\hline P wave variable & Pre-PBMV & Post-PBMV & P-value \\
\hline P-wave maximun & $128.7 \pm 19.6$ & $102.3 \pm 10.2$ & $<0.001$ \\
P-wave minimum & $63.3 \pm 8.6$ & $51.5 \pm 7.1$ & $<0.002$ \\
P-wave dispersion & $65.2 \pm 5.7$ & $50.6 \pm 6.5$ & $<0.001$ \\
\hline
\end{tabular}

Table 5. P-wave variables among patients with cardiac events versus those without events before and after PBMV.

\begin{tabular}{|c|c|c|c|}
\hline Variable & Patients with events $27(31.8 \%)$ & Patients without events $58(68.2 \%)$ & P-value \\
\hline \multicolumn{4}{|l|}{ P-wave maximum } \\
\hline - $\quad$ Pre-PBMV & $130.5 \pm 9.8$ & $119.5 \pm 9.2^{*}$ & $<0.002$ \\
\hline - $\quad$ Post-PBMV & $126.9 \pm 10.1$ & $97.7 \pm 9.6$ & $<0.001$ \\
\hline \multicolumn{4}{|l|}{ P-wave minimum } \\
\hline - $\quad$ Pre-PBMV & $64.1 \pm 6.9$ & $59.7 \pm 5.4$ & $>0.05$ \\
\hline - $\quad$ Post-PBMV & $60.8+5.8$ & $58.9+5.1$ & $>0.05$ \\
\hline \multicolumn{4}{|l|}{ P-wave dispersion } \\
\hline - $\quad$ Pre-PBMV & $66.9 \pm 4.8$ & $54.6 \pm 4.3^{*}$ & $<0.03$ \\
\hline - $\quad$ Post-PBMV & $63.7 \pm 5.2$ & $42.2 \pm 5.0$ & $<0.001$ \\
\hline
\end{tabular}

"Represents $\mathbf{P}<\mathbf{0 . 0 0 2}$. PBMV = percutaneous balloon mitral valvuloplasty.

Table 6. Correlation between P-wave dispersion and pulmonary artery pressure and right ventricular function.

\begin{tabular}{ccc}
\hline Variable & $\mathbf{r}$ & $\mathbf{P}$ \\
\hline PASP & 0.86 & 0.0001 \\
$\mathrm{Em}$ & 0.68 & 0.001 \\
$\mathrm{Am}$ & 0.56 & 0.001 \\
$\mathrm{E} / \mathrm{Em}$ & 0.74 & 0.001 \\
TAPSE & 0.78 & 0.0001
\end{tabular}

PAP $=$ pulmonary artery pressure, Em: early diastolic peak myocardial velocity; Am: late diastolic peak myocardial velocity; E/Em: ratio of early diastolic transtricuspid flow velocity to early diastolic peak myocardial velocity.

value of $86 \%$ and negative predictive value of $96 \%$. $(\mathrm{AUC}=0.913, \mathrm{P}<0.001)$ (Table 7, Figures 1 and 2).

\section{DISCUSSION}

The most striking findings of the present study are:

1) There was significant prolongation of the maximum $\mathrm{P}$-wave duration and $\mathrm{P}$-wave dispersion in patients with MS compared with their age- and sex-matched healthy
Table 7. Cut off values, Sensitivity, specificity, positive predictive value, and negative predictive value of P-maximum and $\mathrm{P}$-wave dispersion in prediction of cardiac events.

\begin{tabular}{ccc}
\hline & $\begin{array}{c}\text { P-wave dispersion } \\
\mathbf{2 6 2 . 8} \mathbf{~ m s e c}\end{array}$ & $\begin{array}{c}\text { P-maximum } \\
\mathbf{\geq 1 1 8} \mathbf{~ m s e c}\end{array}$ \\
\hline Sensitivity & $89.2 \%$ & $85.5 \%$ \\
Specificity & $96.4 \%$ & $91.8 \%$ \\
Positive predictive value & $92.5 \%$ & $86 \%$ \\
Negative predictive value & $98.2 \%$ & $96 \%$ \\
AUC & 0.919 & 0.913 \\
P-value & 0.001 & 0.001 \\
\hline
\end{tabular}

AUC $=$ area under curve.

controls.

2) The maximum P-wave duration and P-wave dispersion in patients with MS had a strong correlation with the degree of pulmonary artery pressure and tissue Doppler indices of right ventricular dysfunction.

3) A significantly higher P-wave dispersion in patients with MS undergoing balloon valvuloplasty, associated with a significant impairment right ventricular functions 


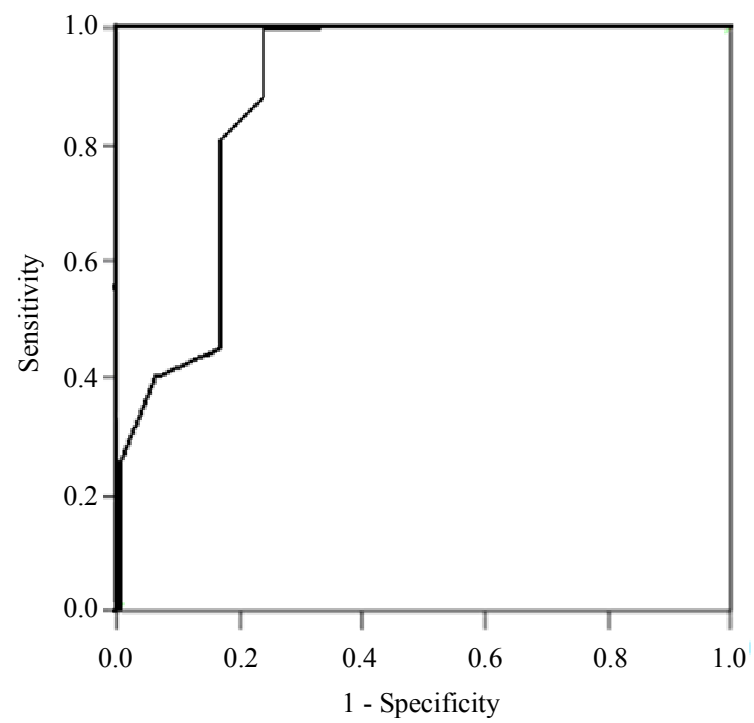

Figure 1. ROC curve for $\mathrm{P}$-wave dispersion $(\mathrm{AUC}=0.919$, $\mathrm{P}<0.001)$.

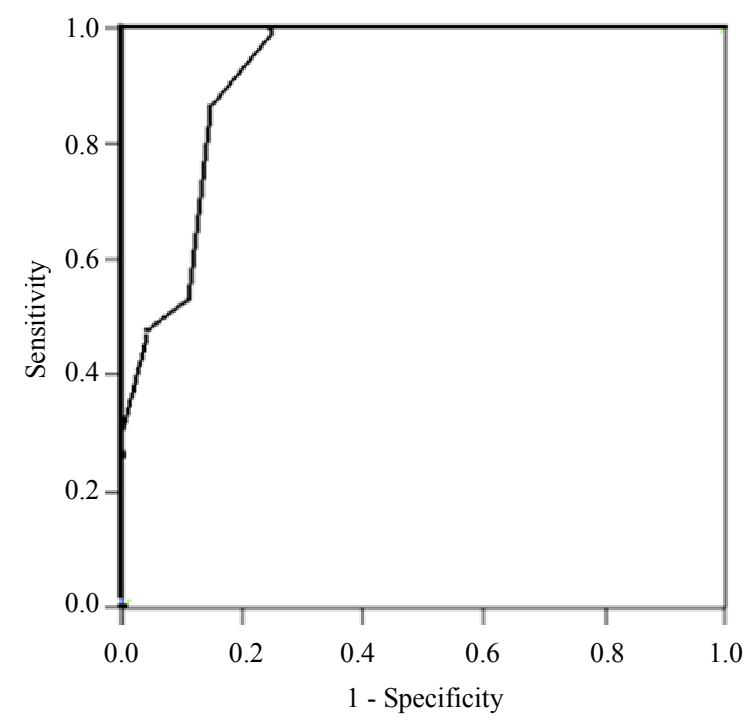

Figure 2. $\mathrm{ROC}$ curve for $\mathrm{P}$ maximum $(\mathrm{AUC}=0.913, \mathrm{P}<$ $0.001)$.

4) Decrease in maximum P-wave duration and P-wave dispersion after PMBV showed a significant correlation with improvement of right ventricular function and regression of pulmonary artery pressure after balloon mitral valvuloplasty

5) P-maximum and P-wave dispersion are independent predictors of cardiac events during follow-up (atrial fibrillation, development of embolic events and recurrent hospitalization) spite of successful PBMV.

6) The cut-of values of P-wave dispersion and Pmaximum in prediction of cardiac events after successful PBMV were $62.8 \mathrm{msec}$ and $118 \mathrm{msec}$ respectively.

Left atrial dilation, fibrosis within the wall of the left atrium and disorganization of the atrial muscle bundles may occur as a result of mitral valve disease and atrial inflammation due to rheumatic carditis. These structural changes can lead to electrical inhomogeneity, nonuniform conduction velocities and inhomogeneous refracttory periods within the atrial myocardium [20], which can be seen on the ECG as increased P-wave duration [21].

There are fewer studies which mention specific changes in the $\mathrm{P}$ wave of electrocardiograms or long term improvement of left atrial enlargement after the procedure. In addition there are no studies in the medical literature which make a correlation between P-wave duration and dispersion before and after percutaneous balloon mitral valvuloplasty (PBMV) and cardiac events after successful PBMV

The current study reports a good correlation of P-wave dispersion with right ventricular dysfunction and increased pulmonary artery systolic pressure in patients with mitral stenosis. In addition significant decrease in $\mathrm{P}$-wave dispersion was associated with significant improvement of RV dysfunction and regression of pulmonary artery pressure during follow-up.

Chandrasekar et al. [22] demonstrated that acute hemodynamic changes following mitral valvuloplasty with a balloon produce changes in the electrocardiogram, indicative of an important hemodynamic benefit resulting from the procedure. In their study patients who had changes in the P-wave patterns had a significant decrease in the left atrium average pressure, smaller residual transmitral gradient and a higher percentage in the opening of the mitral valve. However, the assessment was restricted to the first 72 hours post procedure. Turhan et al. [12] reported that there was a significant decrease in P-wave dispersion following mitral valvuloplasty in both the short and the long term.

During long-term follow-up of patients after successful PMBV, 27 (31.6\%) patients out of 85 patients developed cardiac events 22 patients developed persistent AF, 4 patients became symptomatic (NYHA class III and occurrence of symptoms with pulmonary pressure $>75$ $\mathrm{mmHg}$,), one underwent percutaneous valvuloplasty for restenosis), and two cases developed stroke (associated with recent paroxysmal AF).

The current study showed that patients with cardiac events during follow-up had significantly higher Pmaximum and $\mathrm{P}$-wave dispersion both before and after successful PMBV. Not only this but also the changes of these parameters before and after PMBV were insignificants in patients with cardiac events, while there was a highly significant decrease in P-maximum and P-wave dispersion in patients without late events after PBMV.

Subjects with recurrent AF have been shown to have significantly longer $\mathrm{P}$ wave indices and longer documented history of AF duration compared to individuals 
that maintained sinus rhythm. The largest cohort study included 64 subjects, and found the 28 with recurrence had significantly longer $\mathrm{P}$ wave indices. [23]. Prolonged $\mathrm{P}$ wave indices have been associated with increased risk for incident AF. A retrospective cohort study followed 97 patients with essential hypertension for a mean duration of $25 \pm 3$ months, identifying incident $\mathrm{AF}$ in $20 \%$. 26 Adjusting for age attenuated the odds ratio from 2.09 to 1.34 for prolonged $\mathrm{P}$ wave indices (maximum duration $\geq 130 \mathrm{msec}$ and dispersion $\geq 40 \mathrm{msec}$ ) to predict AF [24]. In a case-control study of 308 hospitalized subjects, the prevalence of $\mathrm{P}$ wave duration $\geq 110 \mathrm{msec}$ was $52 \%$ in the cohort with $\mathrm{AF}$, compared to $18 \%$ of the 308 subjects in a referent cohort. [25] This study had a large age range (26 to 93 years) and calculated $\mathrm{P}$ wave duration from three leads. A nested case-control study from a population-based study found that $70 \%$ of 40 elderly subjects who developed AF after 10 years had baseline $\mathrm{P}$ wave durations $>120 \mathrm{msec}$, compared to $41 \%$ of the 120 controls [26]. $\mathrm{P}$ wave duration remained predictive of $\mathrm{AF}$ following adjustment for covariates of body mass index and hypertension.

\section{CLINICAL IMPLICATION}

Chronic AF is associated with an increased risk of embolic events and negative impact on cardiac function and, therefore, an increased morbidity and mortality risk in patients with rheumatic MS. Therefore, the identification of patients who are susceptible to the development of AF will be of great value. Erbay et al. [27] have shown that four weeks of oral beta-blocker therapy in patients with moderate-to-severe rheumatic MS can significantly reduce the maximum P-wave duration and the P-wave dispersion. Prolongation of maximum P-wave duration and P-wave dispersion may help detect such patients and may be used as an indicator for early intervention in patients with mitral stenosis.

\section{Limitations}

The most important limitation of our study is the manual calculation of $\mathrm{P}$-wave measurements using a magnifying lens instead of computer-assisted P-wave calculations. However the main propose was to investigate the changes of P-wave indices before and after PBMV and whether these non-invasive parameters can predict cardiac events after successful PBMV.

\section{CONCLUSION}

P-wave maximum and dispersion are significantly increased in patients with mitral stenosis and these changes were correlated with significant impairment of right dysfunction and the degree of pulmonary artery pressure. These changes were significantly decreased after suc- cessful PMBV. P-maximum and P-wave dispersion could be considered as independent predictors of late outcome of patients with MS after successful PMBV. The cut-of values of P-dispersion $\geq 62.8 \mathrm{msec}$ and P-maximum $\geq$ $118 \mathrm{msec}$ are highly sensitive and specific in prediction of AF and adverse cardiac events after PBMV

\section{RECOMMENDATION}

This study identifies other simple non-invasive factors that help in prediction long-term results after successful percutaneous balloon valvuloplasty, as P-wave duration and $\mathrm{P}$-wave dispersion. The use of these factors in conjunction with the currently identified predictors allows optimal selection of patients for PMBV.

\section{REFERENCES}

[1] Aytemir, K., Ozer, N., Atalar, E., et al. (2000) P wave dispersion on 12-lead electrocardiography in patients with paroxysmal atrial fibrillation. Pacing and Clinical Electrophysiology, 23, 1109-1112.

doi:10.1111/j.1540-8159.2000.tb00910.x

[2] Gialafos, E.J. (1999) P wave dispersion. A valuable electrocardiographic marker for the prediction of paroxysmal lone atrial fibrillation. Annals of Noninvasive Electrocardiology, 4, 39-45. doi:10.1111/j.1542-474X.1999.tb00363.x

[3] Dilaveris, P.E., Gialafos, E.J., Andrikopoulos, G.K., et al. (2000) Clinical and electrocardiographic predictors of recurrent atrial fibrillation. Pacing and Clinical Electrophysiology, 23, 352-358. doi:10.1111/j.1540-8159.2000.tb06761.x

[4] Boriani, G., Diemberger, I., Biffi, M., et al. (2005) P wave dispersion and short-term versus late atrial fibrillation recurrences after cardioversion. International Journal of Cardiology International Journal of Cardiology, 101, 355-361. doi:10.1016/j.ijcard.2004.03.039

[5] Perzanowski, C., Ho, A.T. and Jacobson, A.K. (2005) Increased P-wave dispersion predicts recurrent atrila fibrillation after cardioversion. Journal of Electrocardiology, 38, 43-60. doi:10.1016/j.jelectrocard.2004.09.008

[6] Palacios, I.F., Sanches, P.C., Harrel, L.C., et al. (2002) Which patients benefit from percutaneous mitral balloon valvuloplasty? Prevalvuloplasty and postvalvuloplasty variables that predict long-term outcome. Circulation, 105, 1465-1471. doi:10.1161/01.CIR.0000012143.27196.F4

[7] Hernandez, R., Banelos, C., Alfonso, F., et al. (1999) Longterm clinical and echocardiographic follow-up after percutaneous mitral valvuloplasty with the Inoue balloon. Circulation, 99, 1580-1586. doi:10.1161/01.CIR.99.12.1580

[8] Iung, B., Cormier, B., Ducimetiere, P., et al. (1996) Functional results 5 years after successlful percutaneous mitral commissurotomy in a series of 528 patients and analysis of predictive factors. Journal of the American College of Cardiology, 27, 407-414.

doi:10.1016/0735-1097(95)00481-5 
[9] Iung, B., Garbaz, E., Michaud, P., et al. (1999) Late results of percutaneous mitral commissurotomy in a series of 1024 patients. Analysis of late clinical deterioration: Frequency, anatomic findings and predictive factors. Circulation, 99, 3272-3278. doi:10.1161/01.CIR.99.25.3272

[10] Orrange, S.E., Kawanish, D.T., Lopez, B.M., et al. (1997) Acturial outcome after catheter balloon commissurotomy in patients with mitral stenosis. Circulation, 95, 382-389. doi:10.1161/01.CIR.95.2.382

[11] Pan, M., Medina, A., Suarez de lezo, J., et al. (1993) Factors determining late success after mitral balloon valvulotomy. American Journal of Cardiology, 71, 1181-1185. doi:10.1016/0002-9149(93)90643-Q

[12] Turhan, H., Yetkin, E., Senen, K., et al. (2002) Effects of percutaneous mitral balloon valvuloplasty on p-wave dispersion in patients with mitral stenosis. American Journal of Cardiology, 89, 607-609. doi:10.1016/S0002-9149(01)02307-4

[13] Marijon, E., Jani, D. and Garbarz, E. (2007) P-wave dispersion and percutaneous mitral valvuloplasty. Cardiology in Review, 15, 42-45.

[14] Dilaveris, P.E., Gialafos, E.J., Sideris, S.K., Theopistou, A.M., Andrikopoulos, G.K., Kyriakidis, M., Gialafos, J.E., et al. (1998) Simple electrocardiographic markers for the prediction of paroxysmal idiopathic atrial fibrillation. American Heart Journal, 135, 733-738. doi:10.1016/S0002-8703(98)70030-4

[15] Steban, A., Alia, I., Tobin, M.J., Gil, A., Gordo, F., Vallverdu, I., Blanch, L., Bonet, A., Vazquez, A., Pablo, R.D., Torres, A., de la Cal, M.A. and Macias, S. (1999) Effect of spontaneous breathing trial on outcome of attempts to discontinue mechanical ventilation. Spanish Lung Failure Collaborative Group. American Journal of Respiratory and Critical Care Medicine, 159, 512-518.

[16] Ghio, S., Recusani, F., Klersy, C., Sebastiani, R., Laudisa, M.L., Campana, C., Gavazzi, A. and Tavazzi, L. (2000) Prognostic usefulness of the tricuspid annular plane systolic excursion in patients with congestive heart failure secondary to idiopathic or ischemic dilated cardiomyopathy. American Journal of Cardiology, 85, 837-842. doi:10.1016/S0002-9149(99)00877-2

[17] Vignon, P., AitHssain, A., François, B., Preux, P.M., Pichon, N., Clavel, M., Frat, J.P. and Gastinne, H. (2008) Echocardiographic assessment of pulmonary artery occlusion pressure in ventilated patients: A transoesophageal study. Critical Care, 12, R18. doi:10.1186/cc6792
[18] Mehta, C.R. and Patel, N.R. (1995) Exact logistic regression: Theory and examples. Statistics in Medicine, 14, 2143-2160.

[19] Hanley, J.A. and McNeil, B.J. (1982) The meaning and use of the area under a receiver operating characteristics (ROC) curve. Radiology, 143, 29-36.

[20] Andrikopoulos, G.K., Dilaveris, P.E. and Richer, D.J. (2000) Increased variance of the P wave duration on the electrocardiogram distinguishes patients with idiopathic paroxysmal atrial fibrillation. Pacing and Clinical Electrophysiology, 23, 1127-1132. doi:10.1111/j.1540-8159.2000.tb00913.x

[21] Braunwald, E. (2001) Valvular heart disease. In: Braunwald, E., Zipes, D.P. and Libby, P., Eds, Heart Disease: A Text book of Cardiovascular Disease, 6th Edition, WB Saunders, Philadelphia, 1643-1653.

[22] Chandrasekar, B., Loya, Y.S., Sharma, S., et al. (1998) Acute effect of balloon mitral valvotomy on serial electrocardiographic changes and their haemodynamic correlation. Indian Heart Journal, 50, 179-182.

[23] Dogan, A., Avsar, A. and Ozturk, M. (2004) P-wave dispersion for predicting maintenance of sinus rhythm after cardioversion of atrial fibrillation. American Journal of Cardiology, 93, 368-371. doi:10.1016/j.amjcard.2003.09.064

[24] Ciaroni, S., Cuenoud, L. and Bloch, A. (2000) Clinical study to investigate the predictive parameters for the onset of atrial fibrillation in patients with essential. American Heart Journal, 139, 814-819. doi:10.1016/S0002-8703(00)90012-7

[25] Agarwal, Y.K., Aronow, W.S., Levy, J.A. and Spodick, D.H. (2003) Association of interatrial block with development of atrial fibrillation. American Journal of Cardiology, 91, 882. doi:10.1016/S0002-9149(03)00027-4

[26] De Bacquer, D., Willekens, J. and De Backer, G. (2007) Long-term prognostic value of p-wave characteristics for the development of atrial fibrillation in subjects aged 55 to 74 years at baseline. American Journal of Cardiology, 100, 850-854. doi:10.1016/j.amjcard.2007.04.017

[27] Erbay, A.R., Turhan, H., Yasar, A.S., et al. (2005) Effects of long-term beta-blocker therapy on P-wave duration and dispersion in patients with rheumatic mitral stenosis. International Journal of Cardiology, 102, 33-37. doi:10.1016/j.ijcard.2004.03.079 